\title{
STUDENT PREFERENCE BETWEEN SINGLE-BOX AND MULTI-BOX HOMEWORK PROBLEM ANSWERS USING WEBWORK, AN ONLINE HOMEWORK SYSTEM
}

\author{
Sayed Mohammad Hashem Jayhooni ${ }^{l}$, Carly Jones ${ }^{2}$ and Agnes d'Entremont ${ }^{2} *$ \\ Departments of ${ }^{1}$ Electrical and Computer Engineering and ${ }^{2}$ Mechanical Engineering, University of British Columbia. \\ *agnes.dentremont@ mech.ubc.ca
}

\begin{abstract}
WeBWorK is an open-source online homework platform used in mathematics as well as engineering, where students can be assigned calculated answer engineering science problems. Problems with staged answers (multi-box) problems are possible on this system, and could offer feedback for the answers at each intermediate step of the solution. This would allow students to determine the step where they had an error (or deficit in understanding), similar to providing a hint on what their specific error was in adaptive feedback systems.

Second-year students in a mechanical engineering program were exposed to both single- and multi-box questions in WeBWorK and were asked to give feedback about their preferences. The vast majority of students reported that they believed that the multi-box questions provided them good feedback on which step or calculation had error(s). They also pointed out the multi-box problems sped up finding errors in their solutions. However, a large minority indicated concern that multi-box problems constrained the solution to a particular path.

Based on these results, providing some multi-box problems may assist students in finding their errors through more detailed feedback on their solution. This may be more effective earlier in a particular topic or in the first problems at any given complexity.
\end{abstract}

Keywords: WeBWorK, online homework system, multibox problems, learning management system, verification feedback.

\section{INTRODUCTION}

WeBWorK is an open-source online homework platform used in mathematics as well as engineering by more than a thousand institutions globally [1]. There are around 35,000 problems, mostly for mathematics courses, created in WeBWorK platform and freely accessible to instructors globally [2]. One of the commonly stated advantages for using online homework is the instantaneous feedback offered by the various systems [3]. This allows students to correct their mistakes in understanding during the process of completing the homework, and not at some later time when solutions are posted.

Two general types of feedback are available in online homework systems - what Huang [3] refers to as verification feedback (right or wrong final answer), and task-specific adapted knowledge of response feedback (referred to hereafter as "adaptive feedback") (suggestions/hints/tutorials based on the specific error that was made).

There is some concern that the simpler verification feedback could lead to trial-and-error strategies that may result in a lost opportunity for student learning. Huang compared these types of feedback in a remedial universitylevel algebra course, and found "no significant differences between two groups in content understanding, problem solving strategies, self-regulation, and achievement" [3].

While verification feedback is fairly simple to implement for calculated answer problems (that is, the solution is calculated based on formulae including variables unique to each student) - the student entered the correct answer or they didn't - adaptive feedback is not as easy to implement - knowledge of a wide range of common mistakes is needed, and the final algorithm may not encompass or correctly map all mistakes.

We suggest that there is a middle ground between the two types of feedback that is nearly as simple to implement as verification feedback for many calculated answer engineering science problems. Often, engineering science problems involve a series of calculations, logical deductions, or steps to arrive at the correct answer. Instead of offering verification feedback only on the final answer, we could offer that feedback for the answers at each intermediate step. This would allow students to determine the step where they had an error (or deficit in understanding), similar to providing a hint on what their specific error was in adaptive feedback systems. We will be referring to this type of feedback as "multi-box" verification feedback (multiple answer entry fields in a single problem), in contrast to "single-box" verification feedback (a single answer entry field in a single problem). 
Generally, students have positive attitudes toward online homework as compared to traditional pen-and-paper homework [4] (for one example). Our previous work has also shown student preferences for one online homework system over another, including around accessibility and clarity of feedback [5]. It is not clear, however, whether students would prefer multi-box or single-box feedback questions in a particular system. With the exception of Huang's work, we have not discovered any research that compared types of feedback in online homework for any outcome measure.

The open-source online homework platform we are using (WeBWorK) has been extensively used in mathematics. Because of its nature as an open-source system, instructors can create their own questions, and the system has substantial flexibility in the number and type of answer boxes included in a single question. Students submit their answers, and feedback (green $=$ correct, red $=$ incorrect) is provided for each individual answer box, allowing students to correct and resubmit their answers. We have converted to WeBWorK from a learning management system based (LMS-based) online homework system where only a single answer was available per question, so we now able to create multi-box questions for the first time in our program. We are interested in determining student preference for either single-box or multi-box questions in WeBWorK.

\section{METHOD}

In order to investigate the effect of multi-box verification feedback, several multi-box questions were created and coded on the WeBWorK platform for second-year mechanical engineering students over two consecutive semesters, as part of a large integrated course (MECH 222) already using WeBWorK for homework. 135 second-year mechanical engineering students were enrolled and completed integrated WeBWorK homework during the term in thermodynamics, fluids and vector calculus.

In addition to their regular homework (single-box problems), 10 multi-box problems, covering thermodynamics topics that spanned the range of content, were created and released to the students on WeBWorK as a study aid prior to final exams. These problems were optional (not assigned for marks), and students could choose whether to complete them. Students were given unlimited attempts per question to reach correct answers (similar to assigned homework) and received immediate feedback on each answer box individually.

The multi-box questions that were coded and uploaded on WeBWorK platform were adapted from a widely-used undergraduate textbook[6], and accessible to all students in the course for around 3 weeks (Fig. 1). Values of specific problem parameters were unique to each student but the method for solving the problem was the same.

Steam is leaving a $3.6 \mathrm{~L}$ pressure cooker whose operating pressure is $150 \mathrm{kPa}$. It is observed that the amount of liquid in the cooker has decreased by $0.52 \mathrm{~L}$ in 36 $\mathrm{min}$ after the steady operating conditions are established, and the cross-sectional area of the exit opening is $7 \mathrm{~mm}^{\wedge} 2$. Determine (a) the mass of steam has leaved the pressure cooker in the period of time, the mass flow rate of the steam and the exit velocity, (b) the total and flow energies of the steam per unit mass, and (c) the rate at which energy leaves the cooker by steam.

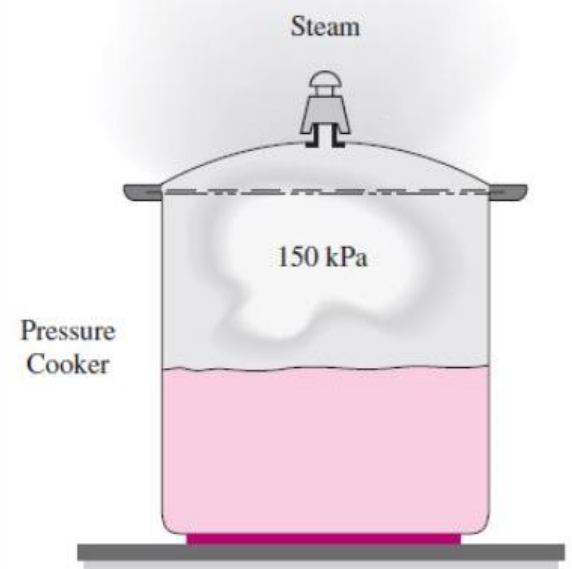

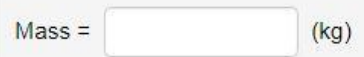
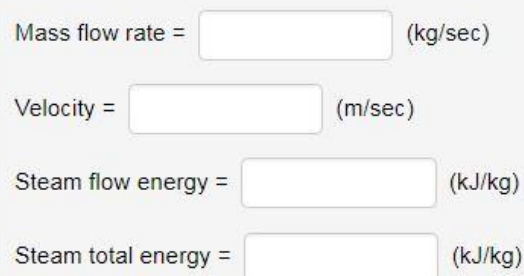

The energy rate leaving the pressure cooker = $(\mathrm{kJ} / \mathrm{sec})$

\# Pay attention to the unit of each parameter

Fig. 1: Sample multi-box question coded and uploaded on WeBWorK platform (question modified from [6]). 


\section{SURVEY AND FEEDBACK}

Students were asked to compare the optional multi-box WeBWorK problems to their assigned single-box homework problems in a survey. An anonymous paper survey was handed out during class time in another course about a week following the final exams of the course using WeBWorK (MECH 222).

Students indicated their agreement (strongly agree, somewhat agree, neutral, somewhat disagree, strongly disagree) with the following statements about their experience with multi-box questions:

1) Multi-box questions provide good feedback on which step/calculation had error(s).

2) Limiting solutions to one particular solution path in multi-box questions are beneficial.

3) Multi-box questions speed up finding errors in my solution.

4) Multi-box questions increase my motivation to solve the problem.

5) Having a guided solution with multi-box questions are very helpful.

6) Multi-box questions are good practice for tests

7) I would like to try more multi-box questions in problem sets

Students could also provide free-form written feedback about multi-box problems on the survey.

\section{RESULTS}

117 students responded to the survey (out of 135 students in MECH 222 - note that two students from MECH 222 were not enrolled in the course where the surveys were given) and 115 students responded to all seven of the statements given above. It should be noted that only 56 students attempted one or more of the optional thermodynamics multi-box problems, so these results combine students responding to our specific problems, and students either predicting their response to these problem, or responding to other multi-box problems they have encountered in other subjects.

Results show that students were generally favourable to multi-box questions (Fig. 2). $88 \%$ of students somewhat or strongly agreed that the multibox questions provided them good feedback on which step or calculation had error(s) (2.6\% somewhat/strongly disagreed, Q1). Similarly, a majority of students believed that multi-box questions sped up finding errors in their solutions (86\% strongly/somewhat agree and $6 \%$ somewhat/strongly disagree, Q3) and that the guided solution provided by multi-box questions was very helpful (83\% somewhat/strongly agree and 4\% somewhat/strongly disagree, Q5).

However, students agreed less often that limiting the solution path was beneficial $(45 \%$ somewhat/strongly agreed and $25 \%$ somewhat/strongly disagreed, Q2). More than half of the students $(54.3 \%)$ stated they strongly or somewhat agreed that multi-box questions increased their motivation to solve the problem $(22.4 \%$ strongly/somewhat disagree with Q4).

$77 \%$ of students express that this type of question is good practice for their tests $(7.7 \%$ somewhat/strongly disagree, Q6) and 76\% of them would like to have more multi-box questions in their problem sets $(4.2 \%$ somewhat/strongly disagreed, Q7).

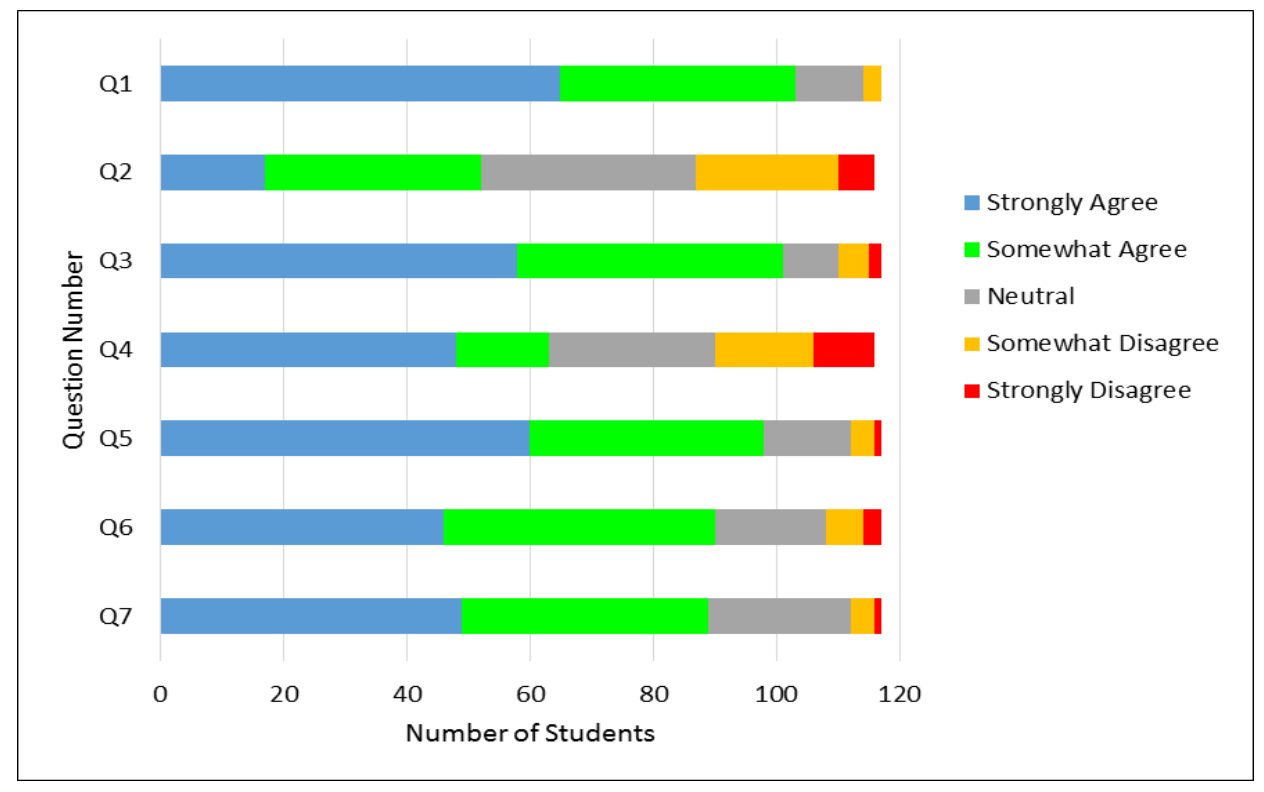

Fig. 2: Students' responses to survey questions. 
Many student comments mentioned that these multi-box questions were helpful. Some also said that this style made the questions clearer and easier to solve. They also pointed out the advantages of guided solutions in this type of question, which provided them the opportunity to solve them step by step as well as being a good learning tool for practicing and understanding, and good preparation for their tests/exams. Quite a number of them requested the creation of more questions and some express that it was a fun and enjoyable method.

However, we have encountered two different views on requiring a particular method (solution path) to approach each question. Some thought it benefits them and liked the idea, and some remarked that sticking to one method is not suitable for all questions; it depended on the particular topic and is different case by case.

Similarly, students' opinions about applying this approach to their assignments or problem sets can be divided to two categories: some of them requested to change all of the single-box questions to multiple-box ones, and others made a request to face both approaches in their future problem sets or assignments.

Finally, a few students reflected that multi-box questions are more intimidating due to the fact that they have more required answers.

We examined the number of times students attempted the problems, for each problem they ultimately fully solved. The average number of attempts was 8.4 (SD 5.4) with a range of 1 to 30 attempts. We compared this with the results from six thermodynamics problems from two other problem sets, which were assigned for marks. In the assigned and graded problem sets, nearly all students attempted the problems. For assigned problems they ultimately solved, students attempted them an average of 3.2 times (SD 5.5) with a range of 1 to 76 attempts.

\section{DISCUSSION}

The survey responses suggest that a majority of students believe that multi-box questions can help to find their source of errors, which may provide better feedback to reduce the likelihood of making those mistakes on tests. The results also suggest that the guided solution may benefit students, but may constrain them to a particular solution path. This may be a benefit when starting to learn a subject. Sometimes students have no idea how to start solving a question and which key parameters are necessary for final requested parameter(s). Therefore, the multi- box problem walks students through step-by-step to final requested parameter(s). The advantage may diminish, however, if they are not given structured problems in tests and exams, if they have relied heavily on the structure of the multi-box problems.

To deal with this drawback, we can create two versions of each question: final answer (single-box) and multi-box. If they are not able to find the right answer in the single-box format, they could be given a chance to switch to the multi-box format for the same question, but would provide feedback that they need more practice prior to the exam.

While many students agreed that the multi-box questions increased their motivation to solve the problem, the response was less positive than to other questions. This may be due to the problems taking more time to fill in (especially getting syntax correct), particularly for some students who already understand the contents very well and are able to solve the related problems quickly, and due to the problems requiring a particular solution path, for students who would like to take a different direction or order of solution. Again, being able to attempt the single-box versions first would give them more freedom to choose the solution path they prefer.

It is unclear how to interpret the data on number of attempts. We expected to see fewer attempts with multi-box questions, because the students would get more specific feedback on errors in the process (assuming they filled all boxes for each attempt). However, because students had unlimited attempts on the questions (as they did for regular homework), we recognize that students may be checking each answer as they go, which would increase the total number of attempts over the single -box problems. As such, this metric is likely not a good proxy for how much time is spent on the problems, or whether students solved the problems more quickly with feedback at each stage. Additionally, while the problems were taken from the course textbook, it is difficult to ensure they were at a similar level to those examined in the problem sets. Further work should be done to evaluate whether multi-box problems reduce the time taken to find a solution.

We suspect it may be simple to convert available single-box questions into multi-box questions, with the addition of the intermediate steps. In some cases, these intermediate values are being calculated in the background already, and simply adding an extra answer box would be required. 
Multi-box verification feedback is not restricted to WeBWorK, so we expect that these results may apply to online homework websites associated with different textbook publishers such as MasteringEngineering [7], WebAssign [8] and McGraw-Hill Connect [9]. The advantages of these sites are their existing problems which usually coordinated with the course text (e.g. problem numbering, notation, et cetera). In addition, the instructors do not need to create or code new problems and those available questions are very well tested and a few of them has errors. The multi-box verification feedback method can easily apply to those available questions and provide suitable feedback for students. However, these online sites are paid sites and students need to register to them to gain access to those questions.

Based on these results, providing some multi-box problems may assist students in finding their errors through more detailed feedback on their solution. This may be more effective earlier in a particular topic or in the first problems at any given complexity.

We are looking forward to applying this method to additional, strategically selected questions for the next academic year.

\section{CONCLUSION}

Students in second-year mechanical engineering program indicated that they believed multi-box problems sped up finding errors in problem solutions. They also believed that the multi-box questions provided them good feedback on which step/calculation had error(s) and also having the guided solutions with this type of questions were very helpful for them. Multi-box verification feedback may lead students to an awareness of what aspect they need to study more and consequently leading to better learning overall.

\section{Acknowledgements}

We would like to take this opportunity to acknowledge Dr. Lacey Samuels and Dr. Luis Linares for all of their support and help as well as students who kindly participated in the survey. We would also like to thank Centre for the Integration of Research, Teaching and Learning (CIRTL) for funding this research.

\section{References}

[1] "WeBWorK Documentation Wiki Main Page." [Online]. Available: http://webwork.maa.org/wiki/Main_Page . [Accessed: 10-Jan-2017].

[2] "WeBWorK Documentation Wiki Open Problem Library." [Online]. Available: http://webwork.maa.org/wiki/National_Pro blem_Library. [Accessed: 10-Jan-2017].

[3] Tzufang (Orchid) Huang. University of Southern California; 2008.

[4] Doorn, David J., Susan Janssen, and Maureen O'Brien. "Student Attitudes and Approaches to Online Homework." International Journal for the scholarship of teaching and learning 4.1, 2010,

[5] Agnes G. d'Entremont, Patrick J. Walls, and Peter A. Cripton, "student feedback and problem development for webwork in a second-year mechanical engineering program," CEEA Annu Conf. - June 4-7, 2017.

[6] Cengel, Yunus A., and Michael A. Boles. Thermodynamics An Engineering Approach (ed.), 2008.

[7] "MasteringEngineering." [Online]. Available: http://www.pearsonmylabandmastering.com/nort $\mathrm{h}$ america/masteringengineering/. [Accessed: 25Apr-2017].

[8] "WebAssign." [Online]. Available: https://webassign.com/. [Accessed: 25-Apr-2017]

[9] "McGraw-Hill Connect." [Online]. Available: http://connect.mheducation.com/. [Accessed: 25Apr-2017]. 